the most interesting. H. pylori is a Gramnegative bacterium, so most of its protein sequences would be expected to resemble other Gram-negative bacteria such as Escherichia coli and Haemophilus influenzae. Most of them do, of course, but a considerable number have sequences that are most similar to other, more distantly related, bacteria. One enzyme that is involved in chorismate biosynthesis is even reported to be most closely related to an equivalent in chloroplasts.

Although the significance of these anomalies is not clear, the possibility of rampant horizontal gene-transfer is unnerving to a community that hopes to reconstruct the history of life on the basis of amino-acid sequence comparison ${ }^{10}$. To test the point, I examined a number of bacterial urease sequences including $H$. pylori (Fig. 2). I found it unsettling not only that the generic boundaries were so ill-defined, but that the plant (jackbean) sequence was almost as similar to each of the bacterial sequences (an average of 65 per cent identity) as most of them were to one another.

There are other frustrations about the data. As in the previous studies, many of the potential gene-encoding regions have not been identified with regard to likely

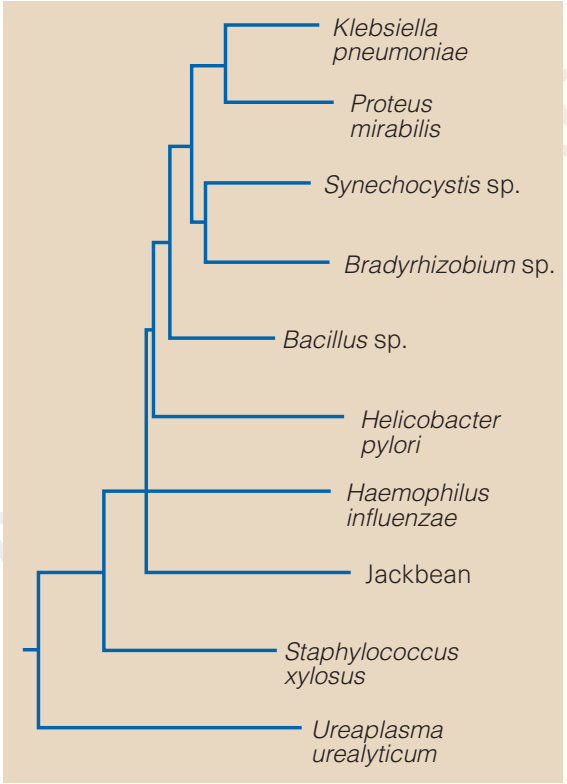

Figure 2 The genome of the Gram-negative bacterium $H$. pylori, sequenced by Tomb et al. ${ }^{1}$, has thrown up a few surprises. One is that certain protein sequences do not most closely resemble those of other Gram-negative bacteria, and this may sound a warning for reconstruction of evolutionary relationships. The phylogenetic tree shown was constructed from a 480-residue, highly conserved region of the enzyme urease. On the average, the plant (jackbean) sequence is 65 per cent identical with the bacterial sequences used. The closest relationship shown is between Klebsiella pneumoniae and Proteus mirabilis, which are 77 per cent identical. function. Given that the genes for all new proteins come from other genes by duplication, in whole or in part, followed by descent with modification, I am surprised that so many open reading frames remain as unidentified reading frames. More rigorous searching will doubtless establish more relationships, as has proved to be the case in the earlier genome reports.

Nonetheless, one cannot help but be impressed by the tremendous accomplishments of Tomb et al. ${ }^{1}$. Not only have they generated the data at a breathtaking pace, but the analyses are both insightful and thorough. The organizational problems associated with searching 1,600 open reading frames against existing databases, and then making proper judgements about the findings, are awesome. Bring on the next bacterial genome sequence - don't hold that TIGR!

Russell F. Doolittle is at the Center for Molecular

Genetics, University of California at San Diego,

La Jolla, California 92093-0634, USA.

1. Tomb, J.-F. et al. Nature 388, 539-547 (1997).

2. Warren, J. R. \& Marshall, B. Lancet 1, 1273-1275 (1983).

3. Marshall, B. \& Warren, J. R. Lancet 1, 1311-1315 (1984).

4. Hugh, T. B. et al. Med. J. Aust. 141, 81-85 (1984).

5. Piper, D. W. Med. J. Aust. 141, 76 (1984).

6. Warren, J. R. Med. J. Aust. 141, 477-478 (1984).

7. Kornberg, H. H. \& Davies, R. E. Physiol. Rev. 35, 169-177 (1955).

8. Vaira, D. et al. J. Clin. Pathol. 41, 355-356 (1988).

9. Censini, S. et al. Proc. Natl Acad. Sci. USA 93, 14648-14653 (1996).

10. Doolittle, R. F. et al. Science 271, 470-476 (1996).

\title{
Asteroids
}

\section{Eros's extended family}

\section{Richard P. Binzel}

ost in space and far from home. That's the fate suffered by the thousands of small asteroids wandering through the inner Solar System near the Earth, cast adrift from the main asteroid belt between Mars and Jupiter by collisions and by Jupiter's gravity. All hope of discovering their heritage would seem to be lost due to the untraceable chaotic evolution of their orbits; and although the unique spectral signatures of some near-Earth asteroids have provided links to their asteroid-belt origins ${ }^{1-3}$, most near-Earth wanderers have no distinctive genealogical traits. But a new approach by Zappalà et al. ${ }^{4}$ has given new insights to the pedigree of the two largest near-Earth asteroids, 433 Eros and 1036 Ganymed $(20 \mathrm{~km}$ and $32 \mathrm{~km}$ across, respectively). They find that Eros and Ganymed may be siblings, originating from a single collision that formed an entire 'family' of small asteroids, most of which still reside in the main belt.

The placement work culminating in the new paper by Zappalà and colleagues was begun seven decades ago by the Japanese astronomer K. Hirayama, who noticed that main-belt asteroids are clustered in their orbital dimensions. Hirayama called these groupings 'families' because he believed their members shared a common origin, and the collisional disruption of asteroid 'parent bodies' is now recognized as the process responsible for creating families.

One family recognized by Hirayama, was called Maria, named after the member with the lowest catalogue number (170 Maria). Orbiting 2.55 AU from the Sun, the Maria family has about 70 identified members, and is precariously poised on the edge of one of the major gaps in the asteroid belt, a 0.05-AU-wide band of unoccupied orbits centred at $2.50 \mathrm{AU}$ ( $1 \mathrm{AU}$ is the mean EarthSun distance). Jupiter probably creates this gap: a circular heliocentric orbit at $2.50 \mathrm{AU}$ has a period exactly one-third of Jupiter's, and any orbit near this 1:3 resonance undergoes chaotic changes in its eccentricity ${ }^{7}$ (becoming more elongated). Eventually, it will have a high enough eccentricity to cross the orbits of the inner planets, so a consequence of this chaotic orbital evolution is the potential for delivering asteroids and meteorites to the inner Solar System from the main asteroid belt ${ }^{8}$.

A critical step in linking Eros and Ganymed to the Maria family was reconstructing the velocity field of bodies emanating from the disrupted parent body. This problem was solved using numerical techniques to determine the orbital parameters of the parent body at the time of the break-up, developed earlier by Zappalà and colleagues ${ }^{9}$. From this velocity field and the size distribution of the recognized family members, the authors now estimate ${ }^{4}$ that on the order of ten objects in the $15-30-\mathrm{km}$ range should have been injected into the 1:3 resonance zone - objects about the size of Eros and Ganymed. It is not clear whether those two objects were the only ones to be successfully injected, or whether their brethren have already met their ultimate fate (collision with the Sun or a terrestrial planet, or gravitational ejection resulting from a close planetary encounter). No other asteroid families are candidates for the heritage of Eros and Ganymed. Zappalà et al. also compare the spectral properties of Eros and Ganymed with those of about a dozen members of the Maria family, and find agreeable matches.

Although the authors make an excellent set of plausibility arguments for the pedigree of Eros and Ganymed, a convincing case is extremely difficult. Injecting such large asteroids into a chaotic zone undoubtedly 


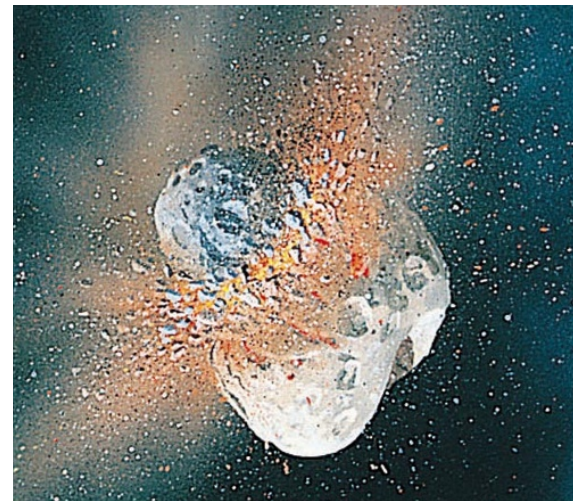

Figure 1 Two asteroids come together to make a family. This artist's impression shows colliding asteroids of different mineralogical types, which would produce meteoritic fragments of varying compositions and some breccias in which fragments of the two types are welded together into a single rock. Such a collision probably gave rise to the Maria family of asteroids, and might indirectly have sent the large near-Earth asteroids Eros and Ganymed into the inner Solar System. (Painting by William K. Hartmann.)

requires a huge collision, but that need not create a recognizable family. Furthermore, Eros and Ganymed have spectral properties that are largely similar to the majority of asteroids in the inner main belt, making their true siblings difficult to recognize.

Despite these difficulties, Zappalà et al. have offered an important new argument. Linking the near-Earth asteroids and their meteorite samples to their original formation locations remains one of the most important challenges for asteroid researchers today. Understanding the family history of Eros is particularly important because this large and accessible Earth-approaching asteroid has been chosen as the destination for the Near-Earth Asteroid Rendezvous (NEAR) mission, which is scheduled to arrive there in 1999. Through observations using NEAR's sophisticated sensors, Eros will become the first asteroid for which we have detailed measurements of chemical composition and elemental abundance. So if we can trace the genealogy and birthplace of Eros, these measurements will establish early Solar System conditions at a known orbital radius within the asteroid belt.

Richard P. Binzel is in the Department of Earth, Atmospheric, and Planetary Sciences, Massachusetts Institute of Technology, Cambridge, Massachusetts 02139, USA.

1. Gaffey, M. J., Reed, K. L. \& Kelley, M. S. Icarus 100, 95-109 (1992).

2. Binzel, R. P. \& Xu, S. Science 260, 186-191 (1993).

3. Cruikshank, D. P., Tholen, D. J., Hartmann, W. K., Bell, J. F. \& Brown, R. H. Icarus 89, 1-13 (1991).

4. Zappalà, V., Cellino, A., Di Martino, M., Migliorini, F. \& Paolicchi, P. Icarus (in the press).

5. Hirayama, K. Jap. J. Astron. Geophys. 6, 137-162 (1928).

6. Hirayama, K. Proc. Imp. Acad. Jap. 9, 482-485 (1933).

7. Wisdom, J. Icarus 56, 51-74 (1983).

8. Wisdom, J. Nature 315, 731-733 (1985).

9. Zappalà, V., Cellino, A., Dell'oro, A., Migliorini, F. \&

Paolicchi, P. Icarus 124, 156-180 (1996).

Wycorrhizal fungi

\section{The ties that bind}

\section{David Read}

$\mathrm{n}$ most higher plants, symbiotic fungi are central to the process of nutrient capture from soil ${ }^{1}$. Evidence from fossils of the earliest land plants ${ }^{2}$, as well as molecular studies $^{3}$, confirms that roots co-evolved with fungal partners to form structures known as mycorrhizas - literally, 'fungus-roots'. These are almost universally distributed through present-day terrestrial plant communities, yet most researchers (deterred, one suspects, from experimental analysis of mycorrhizal function in natural communities by the complexity of these systems) have instead used excised roots or pot-grown plants to examine the relationships between partners in the symbiosis. Unfortunately, reductionist approaches cannot answer larger questions about the effect of symbiosis on interactions between the individual plants that form natural ecosystems.

The study of Simard et al. ${ }^{4}$ (page 579 of this issue) is important in this context. Not only does it address these complex questions in a field situation but, for the first time, it shows unequivocally that considerable amounts of carbon - the energy currency of all ecosystems - can flow through the hyphae of shared fungal symbionts from tree to tree, indeed, from species to species, in a temperate forest. Because forests cover much of the land surface in the Northern Hemisphere, where they provide the main sink for atmospheric $\mathrm{CO}_{2}$, an understanding of these aspects of their carbon economy is essential.

Hyphae are the main structural elements of mycorrhizal fungi. They either penetrate the cells of the plant root to form an 'endomycorrhiza' or, as in most of the trees in the forest studied by Simard et al., they ensheath the root to produce an 'ectomycorrhiza'. In the nutrient-impoverished conditions that prevail in forests, at least 90 per cent of the 'feeding' roots of the tree are colonized by ectomycorrhizal fungi. The result is that a layer of fungal tissue, the mantle, forms an interface between the root and the soil. From this mantle, individual hyphae $(\sim 3 \mu \mathrm{m}$ in diameter) or organized, root-like aggregates called rhizomorphs ( $20 \mu$ m diameter $)$ grow outwards to intimately explore the soil (Fig. 1). Extension of this fungal mycelium into the soil depends on a supply of photosynthetically fixed carbon from the plant ${ }^{1}$. Conversely, essential minerals (especially nitrogen and phosphorus) captured at some distance from the root by the foraging mycelium, are transferred in the reverse direction to the $\operatorname{root}^{1}$.

Fundamentally important for the processes investigated by Simard et al. is the fact that most mycorrhizal fungi are catholic in their choice of host species ${ }^{5}$. As a result, the roots of trees such as the Douglas fir or birch can be colonized by many fungal species, the mycelia of which extend from tree to tree, providing linkages between them. The lack of specificity ensures that, in an undisturbed forest ecosystem, almost all of the trees irrespective of their taxonomic affinities are interconnected by a diverse population of mycelial systems. Groups of tree species joined together in this way have been recognized as functional guilds ${ }^{6}$.

Simard et al. provide a fascinating glimpse of one of the exchange processes facilitated by these underground connections. Using an ingenious approach in which young trees growing close to one another in the forest were simultaneously fed with either ${ }^{14} \mathrm{C}$ - or ${ }^{13} \mathrm{C}$-labelled $\mathrm{CO}_{2}$, they showed that net transfer of carbon occurred from birch to fir, both of which shared up to ten mutually compatible fungal symbionts. Moreover, no such transfer occurred between the species of the ectomycorrhizal guild and cedar, which was colonized by fungi of the endomycorrhizal type. Previous studies ${ }^{7}$ using ${ }^{14} \mathrm{CO}_{2}$ have shown that carbon transfer between plants occurs through the hyphae of compatible mycorrhizal fungi. But these studies could not confirm net flow, because transfer in the reverse

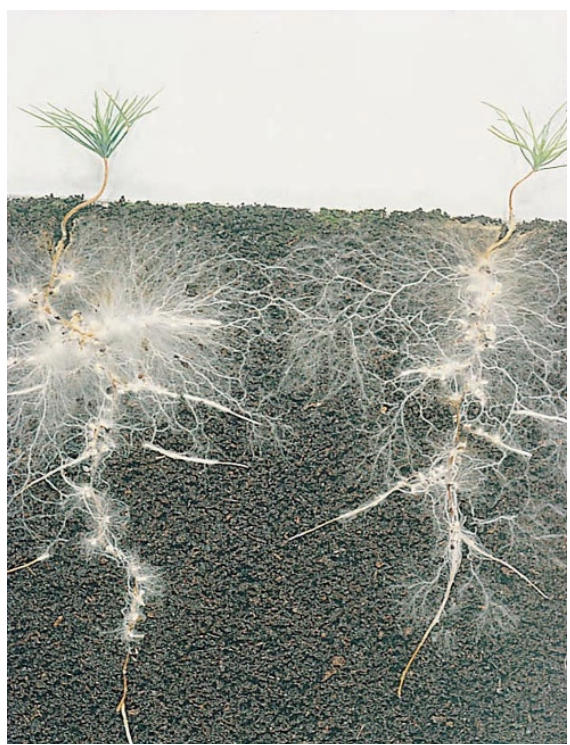

Figure 1 The roots of up to 90 per cent of the trees in a forest can be colonized by many fungal species, the mycelia of which form ectomycorrhizal networks. These networks, shown above linking two conifer species, provide channels for the transfer of nutrients. Simard et al. ${ }^{4}$ have now shown that considerable amounts of carbon can be transferred in either direction between trees through these networks. 